\title{
Estudo através de Espectroscopia de Emissão Óptica do efeito de espécies contaminantes num tratamento por plasma
}

\author{
Plasma treatment contaminant species effect Optical Emission Spectroscopy study
}

Júlio Barbosa $^{1}$; E. S. Medeiros ${ }^{1}$; J. A. B. Oliveira ${ }^{1}$; M. O. Cardoso ${ }^{1}$; A. K. H. Miranda ${ }^{1}$; C. Alves Jr ${ }^{1}$.

\begin{abstract}
Resumo
Espectroscopia de Emissão Óptica (OES) é uma poderosa ferramenta de monitoramento do plasma, com o objetivo de descrevê-lo, pelo menos qualitativamente, e para determinados casos, torná-lo mais eficiente em processos de modificação superficial tais como a nitretação. Especialmente em se tratando de nitretação por plasma, é necessariamente importante que vazamentos do reator de tratamento sejam minimizados para evitar a contaminação do processo devido a espécies indesejáveis da atmosfera. Porém, a capacidade de vedação de cada reator tem um limite o qual não pode ser ultrapassado. Portanto, para reduzir as espécies contaminantes num reator de nitretação, foi estudado o plasma atmosférico em baixa pressão através da OES, em que espécies contaminantes puderam ser identificadas e reduzidas através da adição do gás hidrogênio.
\end{abstract}

Palavras-chave: OES; Plasma; Contaminação.

\begin{abstract}
Optical Emission Spectroscopy (OES) is a powerful tool for monitoring the plasma, in order to (at least qualitatively) describe it. And, in certain cases, to use it more efficiently in surface modification processes such as nitriding. Particularly in the case of plasma nitriding, it is important that leaks in the treatment reactor are minimized to avoid process contamination, due to undesirable species from the atmosphere. However, the sealing capability of each reactor has a limit can not be surpassed. Therefore, to reduce contaminant species in nitration reactor, atmospheric plasma was studied in low pressure conditions, in which contaminating species could be identified and reduced by the addition of hydrogen gas.
\end{abstract}

Keywords: OES; Plasma; Contamination. 


\section{Introdução}

Em qualquer processo a base de plasma, é de vital importância que o sistema conte com o mínimo de contaminação ${ }^{(1)}$ pela presença de impurezas oriundas do gás de trabalho ou mesmo pelo vazamento do sistema de vácuo. Atento a essas possibilidades, é importante conhecer os limites dessa contaminação e, principalmente, eliminá-la do processo. Supondo que todo reator seja devidamente limpo, e as amostras satisfatoriamente preparadas para tratamento, a contaminação por vazamento pode ser estudada e atribuída sua relevância naquele processo ${ }^{(2)}$.

Para tanto, é necessário uma técnica de diagnóstico em tempo real das espécies do plasma. Uma dessas técnicas, que particularmente não interfere na descarga luminescente, portanto caracterizada como não intrusiva, é a Espectroscopia de Emissão Óptica (Optical Emission Spectroscopy - OES). Com ela é possível saber, através do comprimento de onda da luz emitida pelas partículas excitadas que voltaram ao estado fundamental, quais espécies presentes no plasma estão atuando no ambiente de descarga, sejam espécies desejadas ou contaminação ${ }^{(3)}$. Especificamente no processo de nitretação iônica existem várias misturas de gases de trabalho possíveis com o nitrogênio ${ }^{(4)}$, entretanto, comumente utilizar-se a quantidade $20 \% \mathrm{~N}_{2}+80 \% \mathrm{H}_{2}^{(1,5)}$.

Esta proporção corresponde a uma tolerância na quantidade de espécies da contaminação. Sendo assim, estudar a variação do fluxo dos gases permite determinar essa tolerância. Neste trabalho foram realizadas análises espectrais que mostra como reações químicas, baseadas na formação de compostos a partir de combinações das espécies $\mathrm{O}_{2}$ (contaminação gasosa), $\mathrm{H}_{2}$ e $\mathrm{N}_{2}$ (gases de trabalho), podem ser úteis na redução do agente indesejado. Também é determinado que fluxo mínimo da mistura gasosa próprio da nitretação pode ser adotado, para garantir um tratamento adequadamente limpo.

\section{Experimental}

O sistema experimental conta com uma câmara de vácuo cilíndrica montada verticalmente $(30 \mathrm{~cm}$ de diâmetro e $30 \mathrm{~cm}$ de altura confeccionada em aço inoxidável) apropriada para nitretação convencional. Para controle de voltagem no processo, uma fonte de tensão que alcança $1500 \mathrm{~V}$ e corrente máxima 1,5 A. O sistema OES é composto por um espectrômetro Acton Spectrapro 2500i com comprimento focal de $500 \mathrm{~mm}$, resolução espectral de $0.05 \mathrm{~nm}$ e uma rede tripla de difração e um cabo de fibra óptica de $5 \mathrm{~m}$ de comprimento. Neste trabalho foi utilizada a rede de $1200 \mathrm{~g} / \mathrm{mm}$. Como detector, um fotodiodo de silício de $10 \mathrm{~mm}$ de diâmetro responsável pela detecção na faixa de 200-1100 nm. A fibra óptica foi posicionada dentro da câmara, apontando diretamente para descarga luminescente durante todas as medidas, como mostra a Fig. 1.

A varredura espectral se estende da mínima até a máxima capacidade de detecção do sensor.

Para o estudo da descarga luminescente, inicialmente a câmara de tratamento foi evacuada até uma pressão residual de 0,275 mbar. A essa pressão foi ativado o plasma. A primeira coleta de dados foi feita do plasma de gás atmosférico, quando a pressão propositalmente atingiu 1,6 mbar. Em seqüência, foram feitas análises de uma espécie particular presente no primeiro espectro, quando se acrescentou sistematicamente o gás $\mathrm{H}_{2}$, através da variação de seu fluxo. A espécie em questão é um átomo de oxigênio, identificado pelo comprimento de onda de $777,4 \mathrm{~nm}$. Os parâmetros: corrente elétrica e pressão de trabalho foram mantidos constantes, respectivamente 1,6 mbar e 0,05 A. $\mathrm{O}$ fluxo de $\mathrm{H}_{2}$ no estudo variou de 0 até 2 SCCM.

No estudo seguinte, o plasma atmosférico foi monitorado quando a pressão atingiu a pressão de 2,1 mbar. Sistematicamente, o fluxo de hidrogênio foi aumentado de 0 até 2 SCCM, onde o incremento foi mantido em 1 SCCM. Por conseqüência da variação do fluxo, o parâmetro voltagem também experimentou mudança por decrescer, quando os outros parâmetros, a saber, pressão (2,1 mbar), corrente elétrica $(0,22 \mathrm{~A})$ e temperatura do cátodo que é o próprio do porta amostras $\left(452^{\circ} \mathrm{C}\right)$. Dessa vez, o monitoramento foi dirigido ao radical $\mathrm{OH}(306,4 \mathrm{~nm})$.

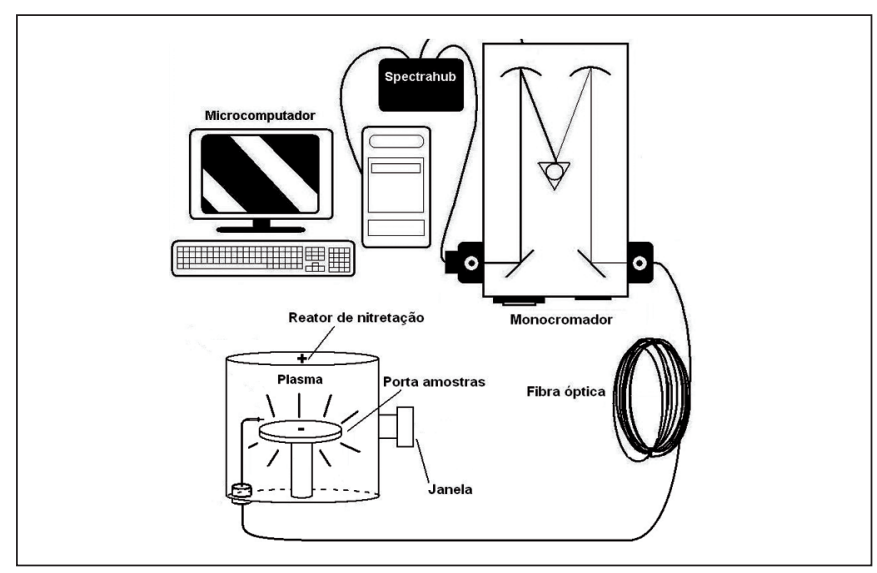

Figura 1. Sistema experimental que consiste em um reator de nitretação e um espectrômetro de emissão óptica. 


\section{Resultados e Discussão}

Com o aumento da quantidade de hidrogênio no plasma, é possível perceber que a primeira espécie presente que reage a este fato é a espécie atômica do oxigênio. A Fig. 2 representa bem esse efeito, já que a intensidade luminosa associada a tal partícula que emite em comprimento de onda de 777,4 $\mathrm{nm}$ decresce à medida que se aumenta $\mathrm{o}$ fluxo de hidrogênio, enquanto que as espécies iônicas e neutras da molécula de nitrogênio permanecem praticamente invariantes.

A Fig. 3 mostra a mesma tendência que outras espécies de oxigênio obedecem, não obstante, nessa figura, a faixa de observação é concentrada no alcance em que os picos mais altos referentes às espécies de nitrogênio não são apresentados.

Apesar de a potência diminuir devido ao decrescimento da voltagem, uma vez que a corrente elétrica é mantida constante, as espécies associadas à molécula de hidrogênio não seguem essa tendência, pois o aumento da pressão parcial deste gás na atmosfera de plasma também cresce. No entanto, apenas a diminuição da voltagem não é o único motivo pelo decréscimo dos picos associados ao nitrogênio e oxigênio. É possível perceber que entre o fluxo 0 a 1 SCCM de $\mathrm{H}_{2}$, há um pequeno aumento do pico associado à espécie de nitrogênio. Isso significa que o pequeno incremento na porcentagem de partículas mais leves no ambiente de plasma promove o aumento da média de energia cinética de elétrons mesmo utilizando menor potência. Isso ocorre porque o gás $\mathrm{H}_{2}$ é mais leve que o gás $\mathrm{N}_{2}$. $\mathrm{O}$ número total de partículas num mesmo volume é menor quanto maior for a concentração do gás menos massivo, mesmo que a pressão de trabalho também seja igual. Menor número de partículas no ambiente de plasma significa maior caminho

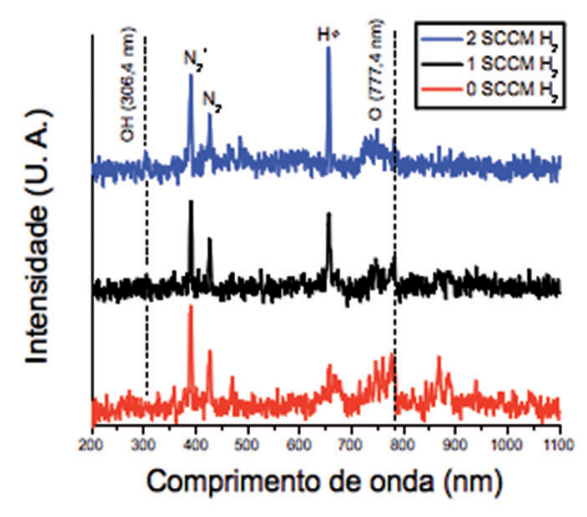

Figura 2. Espectro de emissão de três situações, em que o fluxo de gás $\mathrm{H}_{2}$ varia de 0 até 2 SCCM. livre médio e, portanto maior média de energia cinética de elétrons ${ }^{(2)}$. Com isso, o fato de se aumentar a quantidade de hidrogênio na mistura, formação de moléculas tais como $\mathrm{NH}, \mathrm{OH}$ ou outra combinação de formação de compostos químicos torna-se mais provável minimizando a presença de átomos de nitrogênio e oxigênio.

A Fig. 4 mostra a variação da intensidade luminosa associada á espécie $\mathrm{OH}(306,4 \mathrm{~nm})$ em função da adição do fluxo de hidrogênio no plasma.

Pode-se observar que a cabeça de banda do radical experimenta aumento à medida que as partículas do gás hidrogênio é mais presente, e ao mesmo tempo, os picos associados ao oxigênio decrescem como visto na Fig. 3, indicando que ocorre ligação entre $\mathrm{O}$ e $\mathrm{H}$.

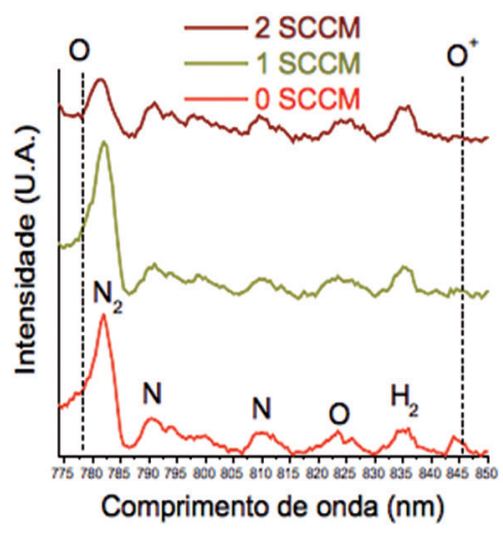

Figura 3. Espectro de emissão de três situações, em que o fluxo de gás $\mathrm{H}_{2}$ varia de 0 até $2 \mathrm{SCCM}$, mantendo constante a pressão $(2,1 \mathrm{mbar})$, corrente elétrica $(0,22 \mathrm{~A})$ e temperatura do cátodo $\left(452^{\circ} \mathrm{C}\right)$.

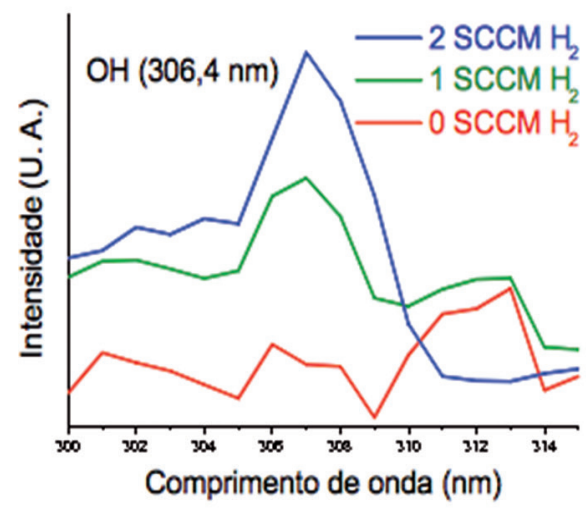

Figura 4. Espectro de emissão relativa à espécie $\mathrm{OH}$ em três situações, em que o fluxo de gás $\mathrm{H}_{2}$ varia de 0 até 2 SCCM, mantendo constante $\mathrm{P}(2,1 \mathrm{mbar})$, i $(0,22 \mathrm{~A})$ e $\mathrm{T}\left(452^{\circ} \mathrm{C}\right)$. 


\section{Conclusões}

Para o vazamento do reator de nitretação do LabplasmaUFRN, foi observado que as espécies de oxigênio proveniente da atmosfera exterior experimentam uma redução significativa quando há adição de hidrogênio no sistema.

Num tratamento de nitretação usual onde a mistura gasosa conta com um fluxo de 2 SCCM de hidrogênio, as espécies de oxigênio se ligam as espécies de hidrogênio tornando-se menos prejudiciais.

\section{Referências}

1. Alves Jr., C., Araújo, F. O., Ribeiro, K. J. B., Costa, J. A. P. Da, Sousa, R. R. M., Sousa, R. S., Use of cathodic cage in plasma nitriding. Surf. Coat. Technol. 201 (2006) $2450-2454$.

2. Barbosa, J. C. P. Análise por meio de espectroscopia de emissão óptica das espécies ativas em nitretação iônica e gaiola catódica. Dissertação de Mestrado (2007). Universidade Federal do Rio Grande do Norte, Natal (RN).
A pequena adição de hidrogênio na mistura de plasma aumenta a sua média de energia cinética de elétrons, porém, essa média não é suficiente para impedir a formação de radicais $\mathrm{OH}$.

\section{Agradecimentos}

Ao CNPq e a Capes pelo apoio financeiro.

3. Fantz, U. et al. Spectroscopy - a powerful diagnostic tool in source development. Nucl. Fusion 46 (2006) S297 - S306.

4. Hirohata, Y., Tsuchiya, N., Hino, T. Effect of mixing of hydrogen into nitrogen plasma. Appl. Surf. Sci. 169-170 (2001) 612 - 616.

5. Souza, S. D., Olzon-Dionysio, M., Miola, E. J., PaivaSantos, C. O. Plasma nitriding of sintered AISI 316L at several temperatures. Surf. Coat. Technol. 184 (2004) 176 - 181. 